Cite this: RSC Advances, 2013, 3, 5798

Received 1st February 2013,

Accepted 20th February 2013

DOI: $10.1039 / c 3 r a 40574 \mathrm{e}$

www.rsc.org/advances

\title{
In situ elastic modulus measurements of ultrathin protein-rich organic layers in biosilica: towards deeper understanding of superior resistance to fracture of biocomposites $\dagger$
}

\author{
Igor Zlotnikov, ${ }^{\text {*a }}$ Doron Shilo, ${ }^{\mathrm{b}}$ Yannicke Dauphin, ${ }^{\mathrm{c}}$ Horst Blumtritt, ${ }^{\mathrm{d}}$ Peter Werner, ${ }^{\mathrm{d}}$ \\ Emil Zolotoyabko ${ }^{e}$ and Peter Fratzl ${ }^{a}$
}

Biogenic ceramics are known to exhibit superior toughness due to a laminated architecture with ultrathin organic layers separating the ceramic blocks. Theoretical analyses relate the toughness increase to the modulus contrast, $E_{\mathrm{c}} / E_{\mathrm{o}}$ between the stiff, $E_{\mathrm{c}}$, and the compliant, $E_{\mathrm{o}}$, components. However, experimental data on this contrast are extremely difficult to obtain by any known technique due to the very small thickness and low modulus values of the organic layers. Here we adapt a recently developed nanoscale modulus mapping technique combined with reverse finite element analysis in order to map the elastic modulus across a $35 \mathrm{~nm}$ thick organic layer within biosilica in a giant anchor spicule of the glass sponge Monorhaphis chuni. We find a modulus of $0.7 \mathrm{GPa}$ in the organic layer as compared to $37 \mathrm{GPa}$ in the bioglass. Furthermore, a modulus gradient extends $50 \mathrm{~nm}$ into the glass layer, probably due to the spatial distribution of small organic inclusions. With this new methodology it becomes possible to determine the elastic moduli of nanometric inclusions even when embedded in a matrix which is $\mathbf{5 0}$ times stiffer.

Superior resistance to the fracture of natural biocomposites, such as nacre of mollusc shells or spicules of marine sponges, has attracted the attention of numerous research groups worldwide as models for tough ceramics. These studies focused on the routes by which organisms drastically enhance (by two to three orders of magnitude) the toughness of brittle ceramics, like calcium carbonate or silica, by adding just a few wt.\% of organic macromolecules. ${ }^{1,2}$ It is commonly accepted that the key point in this evolutionary solution is smart arrangement of alternating layers composed of compliant (organic substance) and stiff

${ }^{a}$ Department of Biomaterials, Max Planck Institute of Colloids and Interfaces, Potsdam 14476, Germany. E-mail: igor.zlotnikov@mpikg.mpg.de

${ }^{b}$ Department of Mechanical Engineering, Technion, Haifa 32000, Israel ${ }^{c}$ Micropaléontologie, UFR TEB, Université P. \& M. Curie, 75252 Paris Cedex 05, France

${ }^{d}$ Max Planck Institute of Microstructure Physics, 06120 Halle, Germany ${ }^{e}$ Department of Materials Science and Engineering, Technion, Haifa 32000, Israel $\dagger$ Electronic supplementary information (ESI) available: sample preparation, modulus mapping, TEM and HAADF-STEM measurements, and finite element analysis. See DOI: 10.1039/c3ra40574e (ceramic) layers, which impedes crack propagation. ${ }^{3-5}$ Earlier works have concentrated on crack deflection and arrest by individual organic-ceramic interfaces. ${ }^{6}$ Structural (and mechanical) inhomogeneities on smaller, that is, nanometer length scales are also known to contribute to the superior mechanical characteristics in a variety of natural biomaterials, such as nacre and bone, ${ }^{7,8}$ biosilica, ${ }^{9}$ or spider silk. ${ }^{10}$ For example, inhomogeneous distributions of $\mathrm{nm}$-sized organic inclusions have been shown by electron tomography and 3D small-angle X-ray scattering in nacre tablets ${ }^{11}$ and in individual calcitic crystallites extracted from specific mollusk shells. ${ }^{12,13}$

Recently, it was demonstrated (by theoretical considerations and model simulations) that the periodic arrangement of compliant and stiff layers is a very important factor, which by itself can dramatically enhance the resistance of biocomposites to fracture. ${ }^{14,15}$ It was shown that the ratio of fracture strength, $\sigma_{\mathrm{c}}$, of a biocomposite composed of the periodically arranged ceramic and organic layers, to the fracture strength, $\sigma_{\mathrm{h}}$, of a homogeneous material (major ceramic component) is defined by the dimensionless parameter:

$$
k=\frac{\sigma_{\mathrm{c}}}{\sigma_{\mathrm{h}}} \approx \sqrt{\frac{a E_{\mathrm{c}}}{2 \lambda E_{\mathrm{o}}}}
$$

where $E_{\mathrm{c}}$ and $E_{\mathrm{o}}$ are, respectively, the elastic moduli of the ceramic and organic components, $a$ is the initial crack length, and $\lambda$ is the spatial periodicity of the alternating layers. It follows from eqn (1) that an increase of fracture strength can be achieved, for example, by decreasing the period, $\lambda$. However, its continuous diminishing strength can hardly be an evolutionary solution since it requires an increasing consumption of organic components, which also leads to the subsequent reduction of the stiffness of the entire structure. A more robust solution, seemingly developed in nature, is to combine the stiff and compliant materials towards producing a high ratio, $E_{\mathrm{c}} / E_{\mathrm{o}}$.

In order to address the question of how high this ratio could be in practice, we have to measure the elastic moduli, $E_{\mathrm{c}}$ and $E_{\mathrm{o}}$, in biocomposites. However, the actual measurement of $E_{\mathrm{o}}$ presents a major challenge, since in many biocomposites the organic 
component is arranged in the form of extremely narrow layers, only a few tens of nanometers in width. Therefore, the mechanical properties of these organic parts, confined within much stiffer ceramic material, and their interaction with the surrounding ceramics remain elusive due to the lack of a suitable experimental approach.

In this work, we apply the nanoscale modulus mapping technique (based on nano-DMA, i.e. nano-Dynamical Mechanical Analysis) to characterize the elasticity of the extremely thin soft layers of a biological composite, the anchor spicule of the glass sponge, Monorhaphis chuni (M. chuni). The latter is composed of alternating amorphous silica and organic layers, as is described in more detail below. This technique was previously used for obtaining high resolution two-dimensional maps of storage and loss moduli in different material systems resolving sub-micronsized structural features with dissimilar elastic and viscoelastic characteristics. For example, in a study of the nacre layer in the shell of a green muscle (Perna canaliculus), organic layers, separating nacre tablets, were clearly resolved in the elastic modulus maps. ${ }^{16}$

The basis of the modulus mapping technique ${ }^{17-19}$ is the wellestablished nanoindentation instrumentation combined with an AFM-like piezoscanner and force modulation system. During scanning, a topographical profile is measured at a constant contact force $F_{\mathrm{DC}}$, which is small enough to avoid tip penetration more than 1-2 $\mathrm{nm}$ deep into the ceramic constituent. Correspondingly, the lateral resolution, which is defined by the contact area of the tip, is estimated to be approximately $10 \mathrm{~nm}$. Due to a tiny penetration depth, the elastic type of contact between the tip and the sample is realized with no plastic deformation involved. For extracting the local stiffness profiles, a modulation force with amplitude $F_{\mathrm{AC}}$, a few times smaller than $F_{\mathrm{DC}}$, is applied to the tip, in addition to $F_{\mathrm{DC}}$. The resultant displacement of the tip is monitored by the piezoelectric transducer. A lock-in amplifier analyzes the transducer's signal and determines its displacement amplitude and phase shift relative to the modulation force signal.

Monorhaphis chuni, used in this research, is a deep sea glass sponge whose habitat is at ocean depths down to 2000 meters. ${ }^{20,21}$ It belongs to the class of Hexactinellida and is among the earliest multicellular animals found as fossils. The most fascinating feature of the M. chuni is the giant basal spicule around which the animal is assembled. This spicule (Fig. 1a) is used for anchoring the animal to the bottom of the ocean and can reach up to $3 \mathrm{~m}$ in length and $8 \mathrm{~mm}$ in diameter. ${ }^{22}$ The organic filament, nearly $2 \mu \mathrm{m}$ in diameter, provides the central vertical axis of the spicule with biosilica cylinders arranged in nearly concentric layers $(2-10 \mu \mathrm{m}$ wide) around it, separated by tiny organic layers (Fig. 1b). Mechanical characteristics of the biosilica inside the spicule have been previously investigated by nanoindentation, with the reduced modulus reported to be $E_{\mathrm{cr}} \approx 42 \mathrm{GPa} .{ }^{22}$ Using the Poisson's ratio, $v \approx 0.3$, yields $E_{\mathrm{c}}=E_{\mathrm{cr}}\left(1-v^{2}\right) \approx 38 \mathrm{GPa}$ for the elastic modulus of biosilica. High efficiency of periodically arranged organic layers to stop crack propagation during indentation was also demonstrated. ${ }^{23}$ However, the mechanical characteristics of the proteinrich organic layers and the organic-ceramic interfaces remained unknown, primarily due to their small dimensions. In fact, the
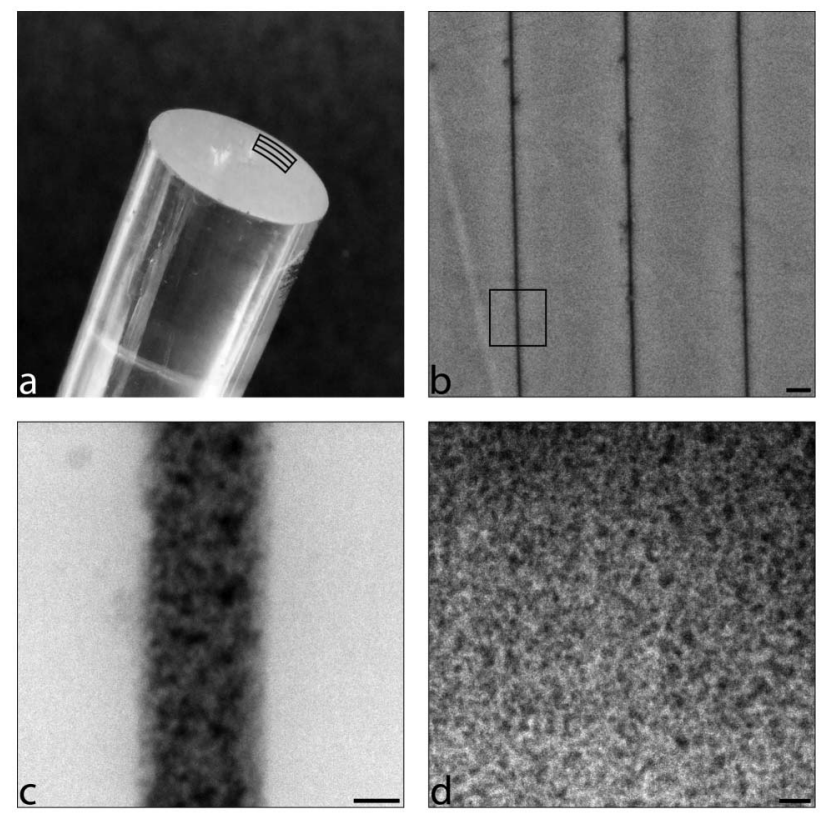

Fig. 1 The structure of $M$. chuni: (a) optical micrograph of the spicule (diameter is 5 $\mathrm{mm}$ ). The organic layers are not visible but their direction is indicated schematically; (b) SEM micrograph of the spicule cross-section (plane view) showing alternating biosilica-organic layers (scale bar is $1 \mu \mathrm{m}$ ); (c) HAADF-STEM image of an individual organic layer (plane-view projection, scale bar is $20 \mathrm{~nm}$ ) taken from the area indicated by the square box in (b); (d) HAADF-STEM image of an individual organic layer (side projection, scale bar is $20 \mathrm{~nm}$ ).

typical width of the organic layer is approximately $35 \mathrm{~nm}$, as is evidenced by high-angle annular dark field (HAADF) imaging in scanning transmission microscopy (STEM) mode (Fig. 1c and d). Gradual contrast variations at the organic-ceramic interfaces, which reflect gradual changes in the organic concentration and therefore modulus variations near the interfaces, are also resolved. In both projections, i.e. in the plane (Fig. 1c) and side views (Fig. 1d), the organic layer does not reveal an extra fiber-like structure.

Organic layers are clearly visible in the nano-DMA maps taken in both the topography (Fig. 2a) and stiffness (Fig. 2b) modes of measurement. Topography profiles of the organic layers reveal shallow grooves 5-10 $\mathrm{nm}$ deep, as a result of a polishing procedure during sample preparation. The presence of these grooves is taken into account later on in stiffness simulations. As expected, experimental stiffness maps show that, in general, the biosilica is much stiffer than the organic layer. The stiffness measured in the biosilica regions can be treated within the Hertz contact model, which is typical to describe elastic interaction of an indenter tip with a homogeneous substrate. ${ }^{24}$ This approach provides a reduced modulus of biosilica $E_{\mathrm{cr}} \approx 44 \mathrm{GPa}$, which fits well with the previously obtained results by nanoindentation. ${ }^{22}$ When in close proximity to the organic-biosilica interface the measured stiffness of the biosilica is significantly decreased. However, in the middle of the organic layer an unanticipated stiffness jump is observed, which appears in the stiffness map as a brighter pass within a much darker surrounding (Fig. 2b). This jump reflects the fact that the tip diameter $(2 R=280 \mathrm{~nm})$ is much 

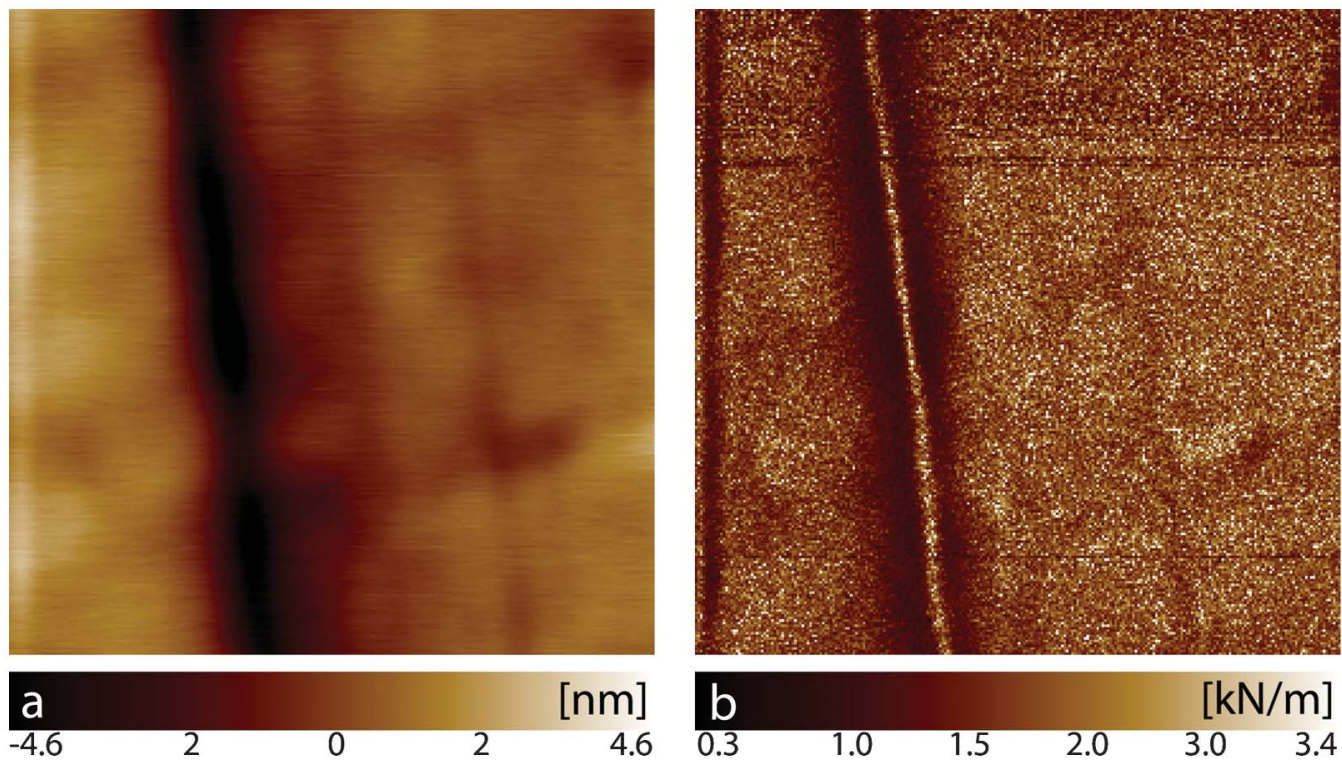

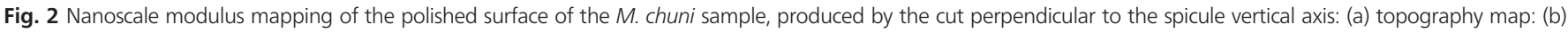
stiffness map. The map size is $1 \times 1 \mu \mathrm{m}^{2}$.

greater than the width of an individual organic layer (35 nm). When approaching the organic layer from the biosilica region during scanning, the tip starts to interact with the softer layer (Fig. 3a) and the measured stiffness is reduced. However, when located in the middle of the organic layer, the tip is maintained by two stiffer biosilica-organic interfaces rather than a soft organic layer (Fig. 3b). Therefore, in this case the stiffness measurement by nano-DMA cannot be interpreted as a direct measure of the elastic modulus of the organic layer, $E_{\mathrm{O}}$ and detailed simulations by finite element analysis (FEA) are required, which are presented in Fig. 3.

Specifically, in order to extract the modulus of the organic part, a numerical replica of the polished cross-section of the spicule was created with a surface topography measured by tapping mode AFM. The width of the organic layer, approximately $35 \mathrm{~nm}$, and the distribution of its concentration in proximity to the interfaces were taken from the HAADF-STEM images, where the contrast strongly correlates with the atomic number of the elements involved and, therefore, with the composition and elastic modules in proximity to the organic-inorganic interfaces. Details of FEA simulations are given in the ESI $\dagger$ and ref. 25.

By using simulated maps we can construct the stiffness profiles across the interfaces and can compare them with experimental ones. We find that the stiffness profiles, calculated at different moduli, $E_{\mathrm{o}}$, reveal specific shapes with a sharp maximum at the center of the organic layer, a minima at some distance away from the center, and an asymptotically growing tail in biosilica regions (Fig. 3c). The calculated stiffness values at the profile center, as well as the profile minima, strongly depend on the modulus of the organic layer, $E_{\mathrm{O}}$. In order to find it, iterative simulations for the abrupt and graded interfaces were performed by varying the value of $E_{\mathrm{O}}$ and the modulus distribution near the interface until a best fit to the experimental profile was achieved (Fig. 3d). The latter yielded $E_{\mathrm{O}}=0.7 \mathrm{GPa}$ and an elastic modulus distribution across the organic layer as shown in Fig. 3e. The extracted value of $E_{\mathrm{o}}$ is in good agreement with the expected elastic modulus of the proteinrich organic material and yields $E_{\mathrm{c}} / E_{\mathrm{o}} \approx 53$. Assuming that the crack length, $a$, in eqn (1) is of the order of period, $\lambda$, we obtain an enhancement factor of $k \approx 5$, which indicates impressive performance of the animal $M$. chuni and a drastic increase of its fracture stress. It is worth mentioning that the measurements were performed on dried samples in ambient conditions. In the natural state of the sponge (i.e. under water), the magnitude of $E_{\mathrm{O}}$ may be lower, which would result in an even higher enhancement factor, $k$.

In conclusion, adapting the nanoscale modulus mapping technique and combining it with iterative finite element simulation allowed determination of the elastic modulus of an organic layer which is only a few tenths of a nanometer thick and confined within a much stiffer biosilica matrix. Previously theoretically postulated structural criteria for small crack stabilization in compliant-stiff layered composites (i.e. significantly lower elastic modulus of the compliant layer and gradation of properties at the interface $)^{15}$ are confirmed in a naturally occurring silica sponge $M$. chuni. Using this methodology, the perspective of measuring the elastic moduli of small and soft inclusions in any nano-composite material becomes accessible.

\section{Acknowledgements}

E. Z. thanks the Shore Research Fund in Advanced Composites for partial financial support of this research. I. Z. thanks the German Science Foundation DFG (Priority Programme SPP 1420). 

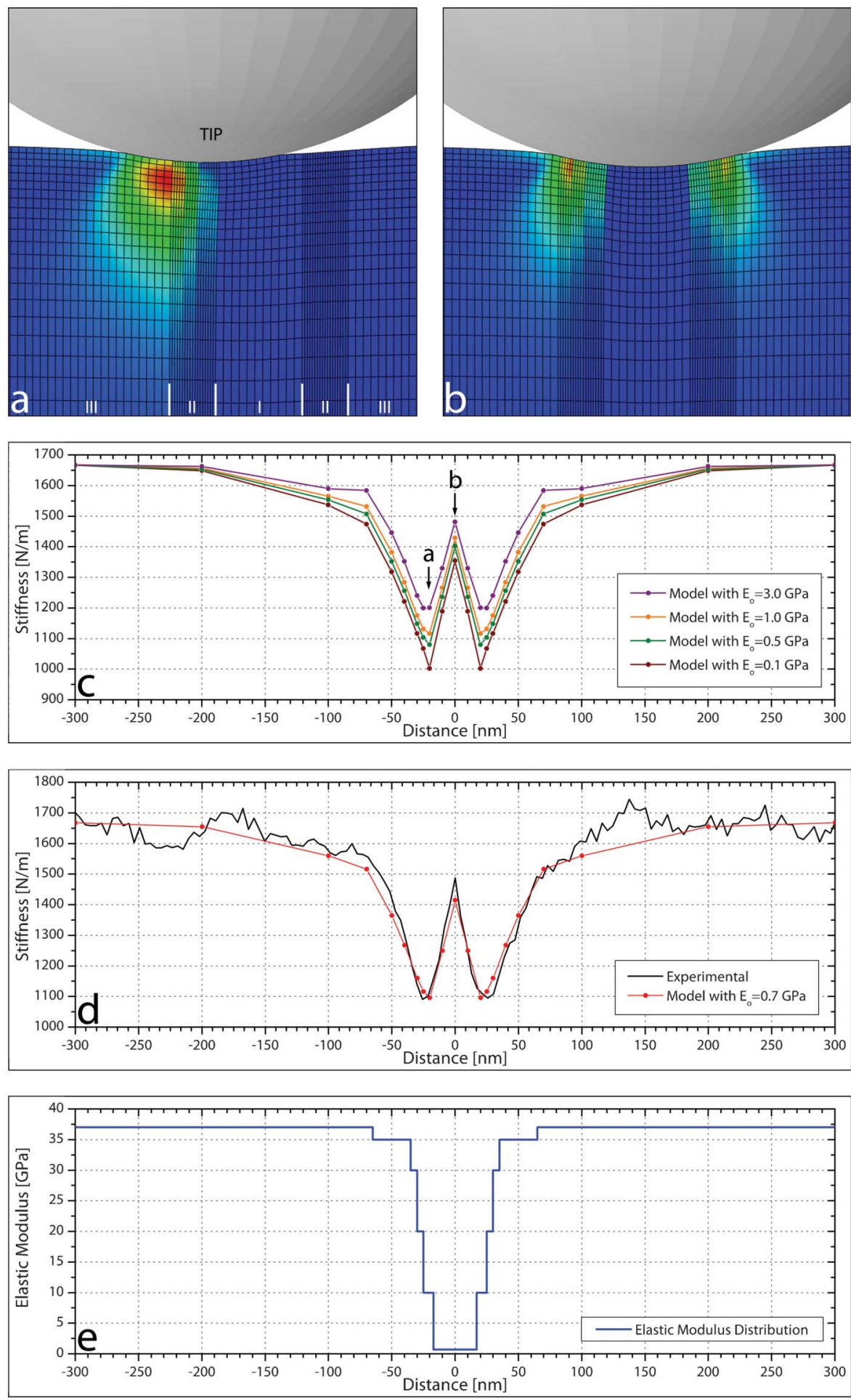

Fig. 3 The calculated and measured stiffness distributions and the resulting modulus distribution in proximity to the organic-ceramic interfaces: (a) a simulated Von Misses stress distribution map when the instrument tip touches only the left edge of the organic layer, at $20 \mathrm{~nm}$ from the center of the organic layer (I - organic layer, II - steep modulus gradient, III - biosilica); (b) a simulated Von Misses stress distribution map when the instrument tip touches both edges of the organic layer; (c) the stiffness profiles across the organic-ceramic interface, calculated at different modules, $E_{0}$, of the organic layer (points $a$ and $b$ on the modulus profiles represent simulated stiffness values for tip positions shown in Fig. $3 \mathrm{a}$ and b, respectively); (d) best fit of the experimental stiffness profile to simulations at $E_{\mathrm{o}}=0.7 \mathrm{GPa}$; (e) elastic modulus distribution across the organic layer that produced a best fit to the experimental stiffness profile. 


\section{References}

1 H. A. Lowenstam and S. Weiner, On Biomaineralization, Oxford University Press, New York, 1989.

2 S. Mann, Principles and Concepts in Bioinorganic Materials Chemistry, Oxford University Press, New York, 2001.

3 H. D. Espinosa, J. E. Rim, F. Barthelat and M. J. Buehler, Prog. Mater. Sci., 2009, 54, 1059-1100.

4 M. A. Meyers, P. Y. Chen, A. Y. M. Lin and Y. Seki, Prog. Mater. Sci., 2008, 53, 1-206.

5 J. W. C. Dunlop and P. Fratzl, Annu. Rev. Mater. Res., 2010, 40, 1-24.

6 H. Ming Yuan and J. W. Hutchinson, Int. J. Solids Struct., 1989, 25, 1053-1067.

7 H. Gao, B. Ji, I. L. Jäger, E. Arzt and P. Fratzl, Proc. Natl. Acad. Sci. U. S. A., 2003, 100, 5597-5600.

8 K. Tai, M. Dao, S. Suresh, A. Palazoglu and C. Ortiz, Nat. Mater., 2007, 6, 454-462.

9 T. Giesa, M. Arslan, N. M. Pugno and M. J. Buehler, Nano Lett., 2011, 11, 5038-5046.

10 D. Sen and M. J. Buehler, Sci. Rep., 2011, 1.

11 S. Younis, Y. Kauffmann, L. Bloch and E. Zolotoyabko, Cryst. Growth Des., 2012, 12, 4574-4579.

12 H. Li, H. L. Xin, M. E. Kunitake, E. C. Keene, D. A. Muller and L. A. Estroff, Adv. Funct. Mater., 2011, 21, 2028-2034.

13 C. Gilow, E. Zolotoyabko, O. Paris, P. Fratzl and B. Aichmayer, Cryst. Growth Des., 2011, 11, 2054-2058.
14 P. Fratzl, H. S. Gupta, F. D. Fischer and O. Kolednik, Adv. Mater., 2007, 19, 2657-2661.

15 O. Kolednik, J. Predan, F. D. Fischer and P. Fratzl, Adv. Funct. Mater., 2011, 21, 3634-3641.

16 H. Moshe Drezner, D. Shilo, A. Dorogoy and E. Zolotoyabko, Adv. Funct. Mater., 2010, 20, 2723-2728.

17 Y. Ganor and D. Shilo, Appl. Phys. Lett., 2006, 88, 233122.

18 S. A. S. Asif, K. J. Wahl, R. J. Colton and O. L. Warren, J. Appl. Phys., 2001, 90, 1192.

19 D. Shilo, H. Drezner and A. Dorogoy, Phys. Rev. Lett., 2008, 100, 035505.

20 C. Levi, J. L. Barton, C. Guillemet, E. Bras and P. Lehuede, J. Mater. Sci. Lett., 1989, 8, 337-339.

21 J. C. Weaver and D. E. Morse, Microsc. Res. Tech., 2003, 62, 356-367.

22 A. Woesz, J. C. Weaver, M. Kazanci, Y. Dauphin, J. Aizenberg, D. E. Morse and P. Fratzl, J. Mater. Res., 2006, 21, 2068-2078.

23 A. Miserez, J. C. Weaver, P. J. Thurner, J. Aizenberg, Y. Dauphin, P. Fratzl, D. E. Morse and F. W. Zok, Adv. Funct. Mater., 2008, 18, 1146-1146.

24 K. L. Johnson, Contact Mechanics, Cambridge University Press, Cambridge, 1985.

25 I. Zlotnikov, H. Drezner, D. Shilo, B. Aichmayer, Y. Dauphin, E. Zolotoyabko and P. Fratzl, MRS Symp. Proceedings, 2011, 1345, 1-10. 